\title{
40 anos da Pós-graduação da EEFE-USP: contribuições para o avanço do conhecimento em Treinamento Esportivo
}

http://dx.doi.org/10.11606/1807-55092017000nesp139

\author{
Valmor Alberto Augusto TRICOLI* \\ Alexandre MOREIRA* \\ Emerson FRANCHINI ${ }^{*}$ \\ Carlos UGRINOWITSCH* \\ Rômulo Cássio de Moraes BERTUZZI* \\ *Escola de Educação \\ Física e Esporte, \\ Universidade de São \\ Paulo, São Paulo, SP, \\ Brasil.
}

\section{Introdução}

Treinamento é caracterizado como um processo repetitivo e sistemático composto de exercícios progressivos que visam o aperfeiçoamento do desempenho. De forma similar, Treinamento Esportivo pode ser definido como um processo sistemático de preparação de atletas para o nível mais alto de desempenho esportivo. Assim, a preparaçáo de um esportista para as competiçóes implica em treinamento organizado com o objetivo de induzir adaptaçóes nos diferentes sistemas do organismo como o muscular, o cardiovascular, o imune, o neural e o metabólico ${ }^{1}$. Essas "adaptaçóes ao treinamento" são frequentemente associadas às alteraçôes no desempenho assim como à determinados fenômenos fisiológicos (protelação do início da fadiga, incremento da potência gerada, etc. $)^{2}$.

No programa de Pós-graduação em Educação Física e Esporte da Escola de Educação Física e Esporte da Universidade de São Paulo (PPGEEFEUSP), os estudos envolvendo os fatores associados à temática "Treinamento Esportivo" foram por muitos anos liderados pelo Prof. Dr. Valdir José Barbanti o qual atuou como orientador de 1982 a 2011. Neste período, o Prof. Valdir formou 24 mestres e oito doutores além de influenciar de maneira decisiva e positiva na formação de um grande número de profissionais dentro e fora do ambiente acadêmico. Os trabalhos orientados por ele investigaram o desempenho e a análise de capacidades motoras e habilidades técnicas em crianças e adultos praticantes de modalidades esportivas individuais e coletivas, a composição corporal de jovens esportistas, os meios utilizados no processo de treinamento e a periodização.

Ainda neste período, mas em particular a partir de 2000, novos docentes começaram a integrar o corpo de orientadores do PPGEEFE-USP, dando continuidade e ampliando as investigaçóes na área do Treinamento Esportivo. Os estudos passaram a abordar temas mais específicos relacionados ao desempenho físico-esportivo, com a preocupação de investigar: a) os efeitos do desenvolvimento de diferentes capacidades motoras; b) a avaliação do processo e o controle da carga de treinamento; c) os modelos de organizaçáo da carga de treinamento; e d) as estratégias mais adequadas para a melhoria do desempenho, todos aplicados a modalidades esportivas de caráter individual e coletivo.

Assim, neste artigo serão relatados os estudos desenvolvidos em nível de mestrado e doutorado junto ao PPGEEFE-USP com destaque para aqueles envolvendo questôes relacionadas à avaliação e o treinamento da capacidade aeróbia, o controle de carga e monitoramento do treinamento, o treinamento da força/potência muscular e o treinamento em modalidades esportivas de combate. 


\section{Avaliação e treinamento em modalidades esportivas de caráter aeróbio}

Tradicionalmente, a compreensão dos fatores determinantes do desempenho nas modalidades esportivas predominantemente aeróbias tem sido objeto de investigação nas Ciências do Esporte. Os primeiros estudos que foram conduzidos no PPGEEFE-USP acerca desses esportes datam do início da década de 1980. Portanto, trata-se de um tema de investigação que possui uma tradição de mais de 35 anos. Algumas informaçóes acerca dos trabalhos que foram conduzidos nas duas primeiras décadas do PPGEEFE-USP podem ser observadas na QUADRO 1. Eles foram, em sua totalidade, orientados pela Profa. Dra. Maria Augusta Peduti Dal'Molin Kiss. A contribuiçáo da Profa. Maria Augusta para o desenvolvimento e a consolidação dessa área não se limitou às orientaçôes dos projetos de pesquisa no PPGEEFEUSP. Ela também participou na estruturação dos primeiros laboratórios de fisiologia do exercício, bem como na formaçáo de recursos humanos que foram fundamentais para nucleação de novos centros de pesquisa em nosso país que atualmente desenvolvem estudos relacionados à aptidáo aeróbia ${ }^{3}$.

QUADRO 1 -Primeiros estudos acerca da aptidão aeróbia conduzidos por alunos regularmente matriculados no Programa de Pós-Graduação da EEFE-USP.

\begin{tabular}{|c|c|c|c|c|}
\hline Ano & Título & Aluno(a) & Nível & Orientador(a) \\
\hline 1983 & $\begin{array}{l}\text { Determinação da condição cardiorrespiratória } \\
\text { dos servidores da Universidade Federal de Mato } \\
\text { Grosso. }\end{array}$ & Anzai K. & Mestrado & Kiss MAPDM. \\
\hline 1988 & $\begin{array}{l}\text { Análise da capacidade aeróbica de atletas } \\
\text { adolescentes. }\end{array}$ & Vallejo Cuellar L. & Mestrado & Kiss MAPDM. \\
\hline 1992 & $\begin{array}{l}\text { Efeito de exercícios aeróbios nos níveis plasmáticos } \\
\text { de 'LP'(a) e lipídeos em sedentários. }\end{array}$ & Todesco D. & Mestrado & Kiss MAPDM. \\
\hline 1993 & $\begin{array}{l}\text { Comparação dos limiares anaeróbio individual e } \\
\text { de lactato analisados pelos testes de laboratório e } \\
\text { de pista. }\end{array}$ & Fleischmann E. & Mestrado & Kiss MAPDM. \\
\hline 1996 & $\begin{array}{l}\text { Efeitos da individualizaçáo da intensidade de um } \\
\text { programa de treinamento contínuo em variáveis } \\
\text { respiratórias e hemodinâmicas de adolescentes } \\
\text { asmáticos graves. }\end{array}$ & Teixeira L. & Doutorado & Kiss MAPDM. \\
\hline 1997 & $\begin{array}{l}\text { Frequência cardíaca em cargas crescentes de } \\
\text { trabalho: ajuste sigmóide, ponto de inflexão e } \\
\text { limiar de variabilidade da frequência cardíaca. }\end{array}$ & Lima JRP. & Doutorado & Kiss MAPDM. \\
\hline 1999 & $\begin{array}{l}\text { Análise das velocidades: referencial de } 4 \mathrm{mM} \text {, } \\
\text { de equilíbrio de } 30 \text { min e velocidade crítica em } \\
\text { nadadoras adolescentes. }\end{array}$ & Colantonio E. & Mestrado & Kiss MAPDM. \\
\hline
\end{tabular}

É interessante observar que uma parte considerável desses estudos objetivou, sobretudo, a análise de diferentes métodos de avaliação da aptidão aeróbia. A escolha dessa linha de pesquisa deve-se ao papel fundamental que o diagnóstico da condição física possui na organizaçấo dos programas de treinamento esportivo e no monitoramento das adaptaçôes fisiológicas que os atletas sofrem ao longo do período de preparação física. Para tanto, implementou-se em meados da década de 1990, entre outras metodologias, a mensuração do lactato sanguíneo em testes progressivos até a exaustão. A ideia central era determinar os limiares metabólicos no intuito de se obter um referencial fisiológico do estresse produzido pelo exercício físico. 
Embora para os padrôes atuais essa abordagem metodológica possa parecer trivial, naquela ocasião era o modelo vigente de pesquisa nessa área. $\mathrm{Na}$ FIGURA 1 podemos contemplar a Profa. Maria Augusta utilizando uma centrífuga, no ano de 1979, ao lado do Prof. Dr. Heinz Liesen da
Universidade de Colônia, Alemanha. Também é possível observar na mesma figura a Profa. Maria Augusta conduzindo um teste progressivo até a exaustão para a determinação dos limiares metabólicos por meio das concentraçóes sanguíneas de lactato.
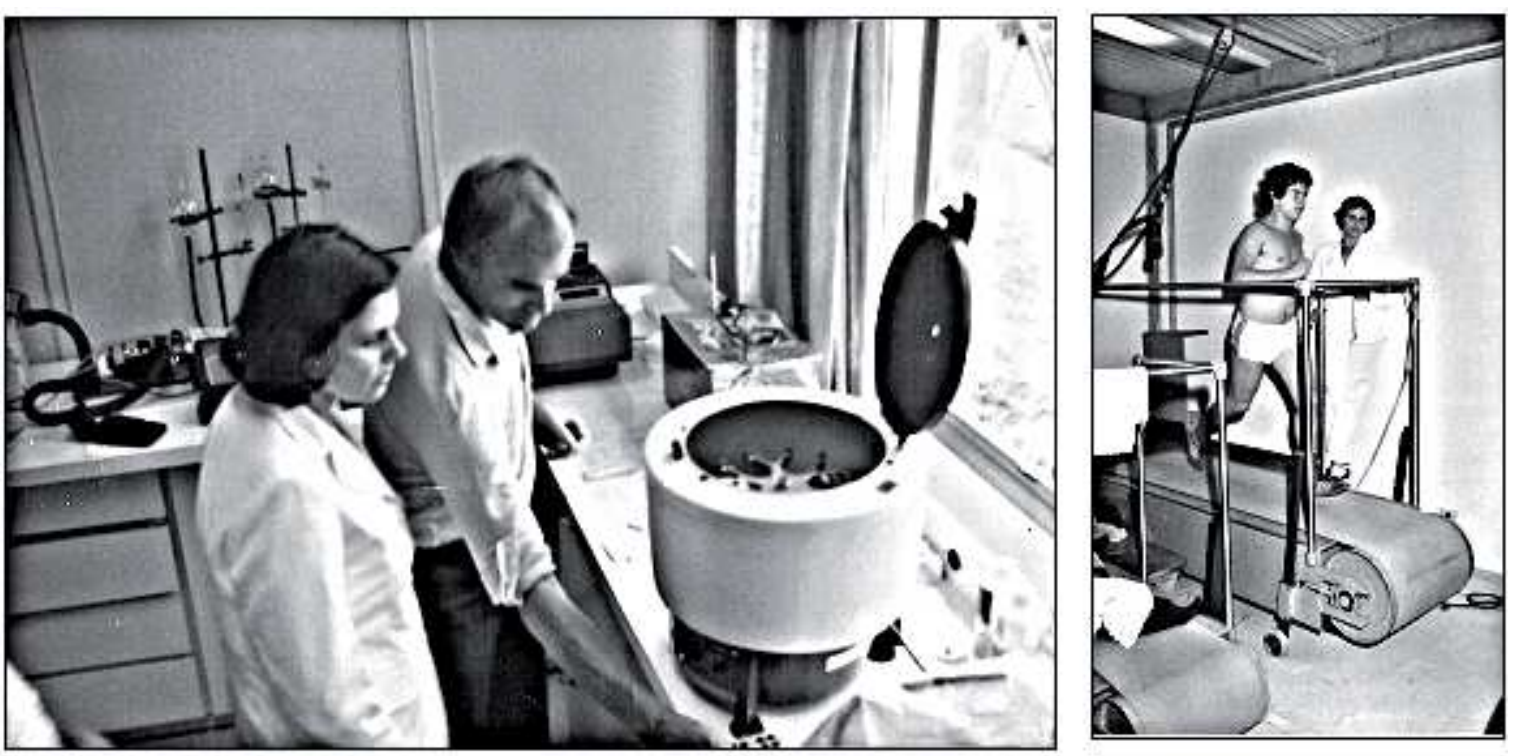

FIGURA 1 - Profa. Maria Augusta ao lado do Prof. Dr. Heinz Liesen (foto à esquerda) e conduzindo um teste progressivo para a determinação dos limiares metabólicos (foto à direita) (fonte: arquivo pessoal Maria Augusta Peduti Dal'Molin Kiss).

Os estudos acerca da análise de diferentes métodos de avaliação da aptidão aeróbia estão sendo desenvolvidos até o presente momento, porém com uma abordagem metodológica contemporânea. Por exemplo, duas dissertaçôes de mestrado foram conduzidas no PPGEEFE-USP com o intuito de compreender a influência dos fatores genéticos sobre a aptidão aeróbia ${ }^{4-5}$.

Com base em achados prévios, tinha-se fortes evidências da importância da genética na aptidão aeróbia. Os achados iniciais demostraram que gêmeos homozigotos tinham valores semelhantes de parâmetros cardiorrespiratórios, como por exemplo, o consumo máximo de oxigênio ${ }^{6}$. Mais recentemente, foi demonstrado que uma série de polimorfismos genéticos poderia estar associada ao desempenho esportivo. No trabalho conduzido por PASQUA ${ }^{5}$ foi analisada a influência de um polimorfismo no gene ACTN3 (R577X) sobre parâmetros associados com a aptidão aeróbia. Através da combinação dos testes ergoespirométricos com a técnica polymerase chain reaction (ou PCR), foi observado que o alelo X conferiu uma vantagem para o alcance do primeiro e do segundo limiares metabólicos em velocidades mais intensas de corrida, o que pode resultar em uma vantagem de indivíduos que possuem esse alelo para o desempenho em provas de longa duraçáo. Já no estudo de BuENo ${ }^{4}$ foi observado que indivíduos com o genótipo II do gene da enzima conversora de angiotensina, o primeiro gene relacionado ao desempenho físico, apresentaram maiores valores do consumo máximo de oxigênio em comparação aos demais genótipos dessa enzima, sugerindo uma influência também desse polimorfismo sobre a aptidão aeróbia. Os interessantes achados dessas duas dissertaçóes podem ser contemplados em artigos recentemente publicados ${ }^{7-9}$.

Além do diagnóstico da aptidão aeróbia, algumas dissertaçóes e teses recentemente defendidas no PPGEEFE-USP foram conduzidas no intuito de compreender o impacto de diferentes regimes de treinamento sobre a melhora do rendimento em esportes de longa duração. Em um desses trabalhos foi 
investigada a influência do treinamento intervalado de alta intensidade sobre variáveis fisiológicas e o desempenho de corredores em teste contrarrelógio de $5 \mathrm{~km}^{10}$. Foi observado que o treinamento promoveu uma melhora de quase $8 \%$ na economia de corrida, mas não modificou o desempenho dos corredores no teste contrarrelógio de $5 \mathrm{~km}$. Isso significa que, embora o treinamento tenho melhorado uma variável fisiológica tradicionalmente relacionada à aptidão aeróbia, essa melhora não resultou em maior rendimento atlético. Provavelmente, isso se deve ao fato de outros fatores fisiológicos serem mais importantes para o sucesso nesse esporte. Em outro estudo foi analisado o impacto de um programa de treinamento de força sobre a estratégia de prova adotada por corredores em um teste de contrarrelógio de $10 \mathrm{~km}^{11}$. Os resultados mostraram que os atletas submetidos ao regime de treinamento melhoraram o desempenho, sobretudo por conseguirem percorrer o último terço do teste em intensidades mais elevadas. Esses achados sugerem que corredores fundistas que têm dificuldade de sustentar altas intensidades próximo ao final da prova podem se beneficiar do treinamento de força. Maiores detalhes desse estudo podem ser contemplados em um artigo publicado por DAMASCEno et al. ${ }^{12}$.

Nesse sentido, pode-se observar que ao longo dos seus 40 anos de existência o PPGEEFE-USP têm contribuído de forma expressiva tanto para o avanço do conhecimento de temas que envolvem a aptidão aeróbia quanto para a atuação profissional, haja vista que uma parcela considerável de profissionais do esporte utilizam a avaliação e o treinamento físico em suas intervençóes.

\section{Controle de carga e monitoramento do treinamento}

O desafio na organização do conteúdo de treinamento é determinar a relação ideal entre dose e resposta, evidenciando assim a importância de se planificar, quantificar, e buscar o monitoramento regular e sistemático do estresse (carga) de treinamento e competição, das respostas adaptativas advindas do processo de preparação (fadiga e condicionamento), e das alteraçóes resultantes de desempenho. Cada sessão de treinamento ou competição impóe ao atleta um estresse fisiológico ${ }^{13}$. Como em todas as formas de estresse fisiológico, há uma reação homeostática, que, por sua vez, resulta em alteraçóes metabólicas e fisiológicas transitórias ${ }^{14}$ cuja magnitude parece ser decorrente e dependente da combinação entre demanda física e emocional ${ }^{15-16}$. BuORDON et al. ${ }^{17}$ afirmam que o monitoramento da carga de treinamento é essencial para determinar se (e como) os atletas estáo se adaptando ao programa de treinamento; avançar no entendimento das respostas individuais, e avaliar a fadiga e a necessidade "para a recuperação", minimizando o risco de um "overreaching" não funcional, lesôes e infecçôes.

Considerando o anteriormente exposto o Grupo de Estudos e Pesquisa em Planejamento e Monitoramento do Treinamento Físico e Esportivo tem se preocupado em contribuir com a produção de conhecimento, notadamente no que se refere a organização e monitoramento do processo de treinamento, as relaçóes entre estresse e infecçóes e carga de treinamento, respostas neuro-imuno-endócrinas agudas e crônicas no treinamento esportivo, e análise do desempenho, centrada tanto no indivíduo quanto no grupo (esporte coletivo). Assim, nesta seção, seráo apresentados os principais trabalhos conduzidos pelo Grupo nos últimos 10 anos junto ao PPGEEFE-USP.

Estudos têm sido realizados para examinar as respostas agudas de sessóes de treinamento e competiçáo, para uma melhor compreensão dos fatores que podem influenciar na associação entre o estresse da competição ou treinamento e psicofisiológicas, em especial, na carga interna, parâmetros hormonais e imunológicos. Por exemplo, a resposta do cortisol salivar e da imunoglobulina salivar A foi investigada em jogadores profissionais de futebol durante jogo simulado ${ }^{18-19}$. Os resultados estimularam estudos com delineamentos semelhantes, com outras modalidades esportivas coletivas e de combate, a fim de melhor compreender os fatores associados às respostas imuno-endócrinas e comportamentais em treinamento e competição. Assim, as análises foram estendidas (jogo simulado, respostas pré-pós competição) em jogadores de alto nível de futsal ${ }^{19}$ e de basquetebol ${ }^{20}$. Como consequência desses projetos, a comparação entre as respostas psicofisiológicas de atletas em situação de competição oficial e simulada fez-se necessária, para que a restrição ambiental fosse contemplada. Investigações com essa abordagem foram realizadas 
com atletas de basquetebol profissional ${ }^{21}$ e jovens ${ }^{22}$, lutadores de jiu-jitsu ${ }^{23}$ e jovens jogadores de futebol de campo ${ }^{24}$. A importância de uma competição e o efeito psicofisiológico de se competir "em casa" e "fora de casa" também foram alvo de investigação no voleibol ${ }^{25}$, no basquetebol ${ }^{26}$ e no futsal ${ }^{27}$.

As respostas agudas e crônicas ao treinamento, considerando os métodos frequentemente utilizados por treinadores, também necessitam ser melhor compreendidas a partir de evidências empíricas para que possibilitem uma prática mais fundamentada. Assim, um dos métodos de treinamento que tem sido amplamente utilizado por profissionais nas modalidades esportivas coletivas, tem sido alvo de investigação do Grupo: o jogo reduzido. No estudo de Marcelino et al. ${ }^{28}$ buscou-se observar o efeito da manipulação da dimensão da área do jogo, nas respostas metabólicas, perceptuais e de desempenho em jogadores de basquetebol. De forma semelhante, no futebol, a análise do desempenho técnico e físico durante os jogos reduzidos também foi alvo de investigação abordando as alteraçóes temporais ao longo dos jogos reduzidos, e as possíveis associaçôes entre desempenho técnico e físico, e a fadiga transitória ${ }^{29}$. Adicionalmente no trabalho de Rodrigues Lopes ${ }^{30}$ a análise de jogo, notadamente para o desempenho técnico, tático e físico nos jogos reduzidos, foi realizada no sentido de acompanhar as possíveis alteraçôes individuais e de padrão coletivo, ao longo de 14 meses, em jovens jogadores de futebol. Além disso, buscou-se verificar o efeito das medidas de desempenho físico e antropométricas, nessas respostas.

A dinâmica da carga de treinamento e as respostas ao longo da preparação dos atletas é outro tema que também tem sido investigado pelo Grupo. Por exemplo, nos estudos de Moreira et al. ${ }^{31-32}$ foi descrito e analisado o conteúdo da periodização do treinamento em jogadores de futebol australiano e de rugby, respectivamente. A carga interna de treinamento e a intensidade percebida pelos atletas em diferentes tipos de sessóes de treinamento ao longo das respectivas temporadas foi considerada. A demanda competitiva para jogadores de diferentes posiçóes, bem como a influência da organização temporal das cargas em microciclos distintos foram examinadas nestes estudos.

Para além de estudos longitudinais exploratórios, é fundamental a condução de pesquisas que contemplem a manipulação experimental das cargas de treinamento. Uma das estratégias que tem sido amplamente utilizada no esporte é a de intensificação (e "tapering") das cargas de treinamento ${ }^{33}$. Este aspecto também tem sido foco de estudo em modalidades como futebol ${ }^{34}$, basquetebol $^{35-41}$, futsal ${ }^{42}$, tênis ${ }^{43}$ e ginástica rítmica ${ }^{44}$. Os resultados destes estudos vêm contribuindo para um melhor entendimento acerca dos efeitos dessa estratégia de periodização do treinamento. Nessas investigaçóes, os resultados têm sugerido que a percepção subjetiva de esforço da sessão (PSE) é um método válido e útil para a quantificação e monitoramento do treinamento, sendo sensível à manipulação das cargas de treinamento e competição, em diferentes modalidades esportivas e populaçóes; e que a adoção de instrumentos psicométricos para o acompanhamento e análise das respostas comportamentais “associadas” à manipulação da carga, tais como os estados de humor, bem-estar, tolerância ao estresse, fadiga percebida, percepção da recuperação, e sinais e sintomas relacionados os riscos de infecçóes, pode colaborar com a eficácia do monitoramento do treinamento. Um importante aspecto a se destacar é a proposição do Grupo concernente à possibilidade de implementação da estratégia de intensificação e "tapering", baseada na concentração das cargas de força, em particular, para modalidades coletivas, em jogadores adultos e jovens ${ }^{35,38-40}$.

A importância do monitoramento longitudinal em jovens atletas, também foi demonstrada no estudo de Moreira et al. ${ }^{45}$. Neste estudo as respostas da imunoglobulina salivar A e da severidade de infecçôes do trato respiratório superior (ITRS), foram investigadas em jovens jogadores de futebol $(\sim 13$ anos de idade). Os resultados revelaram a necessidade de se monitorar a carga de treinamento e as alteraçóes comportamentais, no sentido de minimizar a ocorrência e severidade dos sintomas de ITRS e o comprometimento da função da imunidade da mucosa oral, consistente com o verificado em estudos com atletas adultos ${ }^{46}$.

Em outro estudo longitudinal, sem intervenção, com jovens jogadores de futebol (12,5 anos), ficou demonstrado o papel modulador da concentração de testosterona no desempenho de testes físicos ao longo da temporada ${ }^{47}$. A dinâmica da resposta da testosterona em jovens jogadores (sub-15 e sub17) também foi observada em estudo do Grupo ${ }^{48}$, em conjunto com as alterações de desempenho ao longo da temporada. Estes resultados sugerem a importância de se monitorar (e considerar) o "status" hormonal de jovens atletas, na avaliação do desempenho e das alteraçóes comportamentais, e se alinham com os resultados observados com jogadores de voleibol (sub-16 e sub-19) ao longo de dois macrociclos de preparação ${ }^{49}$, os quais indicam 
que o comportamento parece ser mais afetado nos jogadores mais jovens.

A análise do desempenho (análise de jogo) e o efeito nos diferentes parâmetros, comportamentais, carga interna, carga externa, respostas hormonais, imunes e perceptuais, durante competições nas quais jovens jogadores são submetidos a jogos sucessivos em períodos relativamente curtos de tempo, também tem sido pesquisado. Esses tipos de competição ocorrem com frequência e são disputadas tanto em nível nacional quanto internacional ${ }^{50}$. Em um dos estudos $^{51}$, durante um campeonato internacional de futebol (sub-15), foi demonstrada a sensibilidade das medidas de aceleração e impacto, notadamente, no que se refere à identificação do efeito dos jogos sucessivos sobre o desempenho físico. Considerando a necessidade de uma abordagem holística e integrada para a análise do jogo no futebol ${ }^{50}$, buscou-se verificar as alteraçóes do desempenho técnico, as respostas hormonais e imunes, de jogadores participantes de um campeonato com jogos sucessivos (sete jogos em sete dias $)^{52}$. Os resultados desse estudo são bastante promissores e mostram uma dinâmica de queda da testosterona ao longo da competição, sugerindo um estado de fadiga acumulada, em concomitância com o comprometimento das açóes defensivas, sem alteração na percepção do esforço; o que sugeriria um ajuste dos jogadores no tocante às suas açóes e esforço durante a atividade, a fim de preservar o equilíbrio entre a demanda da atividade e seu gasto energético, considerando as possibilidades "atuais" (estado momentâneo) do atleta, no sentido de concluir o jogo (finalizar a atividade).

Em resumo, os resultados dos estudos conduzidos pelo Grupo de Estudos e Pesquisa em Planejamento e Monitoramento do Treinamento Físico e Esportivo nos últimos 10 anos têm sugerido a necessidade de um monitoramento integrado de indicadores comportamentais, fisiológicos, perceptuais, imunológicos, físicos, técnicos e hormonais. A análise das respostas dos atletas ao treinamento e competição; indicam também, a importância de se considerar as restriçóes individuais e de ambiente para determinar o efeito da carga competitiva e avançar o conhecimento acerca da análise de desempenho (análise de jogo), no contexto do planejamento e monitoramento do treinamento físico-esportivo.

\section{Treinamento da força/potência muscular}

O Grupo de Estudo e Pesquisa em Adaptaçóes Neuromusculares ao Treinamento de Força vem desenvolvendo pesquisas sobre os efeitos do treinamento de força/potência, em diferentes contextos, ao longo dos seus 16 anos de existência. Em um desses contextos, objetivamos entender como diferentes métodos e modelos de treinamento de força afetam o rendimento esportivo em diferentes níveis; contudo, há diversos problemas quando tentamos estudar atletas, sendo o principal deles expô-los a riscos desnecessários. Por isso, vários dos estudos do Grupo foram feitos com jovens treinados e/ou fisicamente ativos, mas não necessariamente atletas. Esses estudos foram realizados tendo quatro objetivos básicos: investigar a) os efeitos de diferentes métodos e/ou cargas de treinamento de força no rendimento de testes motores; b) os efeitos de diferentes modelos de organização da carga do treinamento de força no rendimento de testes motores (e.g. agilidade, velocidade, salto vertical, força máxima, potência de membros inferiores, etc.); c) os efeitos de diferentes métodos de treinamento de força na coordenação e no controle de habilidades motoras básicas (e.g. salto vertical e corrida); e d) os efeitos do treinamento de força em ações técnicas e táticas esportivas.

Considerando os efeitos de diferentes métodos e/ ou cargas de treinamento de força no rendimento de testes motores, em 2005 TRICOLI et al. ${ }^{53}$ comparam os efeitos de duas combinaçóes de treinamento de força: treinamento tradicional + levantamento olímpico (LPO) vs. treinamento tradicional + pliometria. Esse estudo mostrou que a primeira combinação (treinamento de força tradicional + levantamento olímpico) foi muito mais efetiva do que a segunda em aumentar o rendimento em vários testes motores. Esse trabalho foi considerado seminal à época e hoje pode ser considerado um clássico na área específica do treinamento com LPO. Ainda procurando verificar os efeitos de combinaçóes de diferentes métodos de treinamento, KoBAL et al. ${ }^{54}$ verificaram se a ordem com que o treinamento de força e o treinamento pliométrico são realizados dentro das sessóes de treinamento (1. 
força seguido de pliometria; 2 . pliometria seguida de força; e 3. séries alternadas de força e pliometria) afetava a melhora do rendimento em testes motores. Ao final do período de treinamento, as diferentes ordens adotadas nas sessões aumentaram de maneira similar o rendimento nos testes motores. A única ressalva foi que a sequência 2 (pliometria seguida de força) apresentou maior aumento da velocidade de corrida do que as outras combinaçóes.

O Grupo também investigou os efeitos crônicos e agudos de vários métodos de treinamento no rendimento em testes motores. LAMAs et al. ${ }^{55}$ procuraram identificar as diferenças nas adaptaçóes causadas pelo treinamento de força de alta intensidade e pelo treinamento de potência e verificaram que ambos os métodos foram efetivos em aumentar a força e a potência de maneira similar nos membros inferiores de indivíduos. Já BATISTA et al. ${ }^{56}$ testaram o efeito de contraçóes musculares intermitentes no desencadeamento da potencialização pós-ativação (PPA) e o consequente aumento na produção de força enquanto LAMAs et al. ${ }^{57}$ verificaram a ocorrência da PPA com o uso de exercícios realizados em plataformas vibratórias. Ambos estudos não observaram a manifestação da PPA e comprovaram que os exercícios utilizados não foram eficientes em aumentar a produçáo de potência muscular e o desempenho no salto vertical. Devido à alta variabilidade na ocorrência da PPA, BATisTA et al. ${ }^{58}$ expandiram as hipóteses anteriormente testadas de que o nível de treinamento dos indivíduos afetava a ocorrência da mesma. Neste estudo, os achados indicaram que o nível de treinamento em força e/ou potência náo afetava a ocorrência da PPA, porém alguns indivíduos apresentaram aumentos de rendimento, enquanto outros não. Assim, protocolos de treinamento baseados na PPA devem ser utilizados apenas por indivíduos que sejam responsivos a esta estratégia de treinamento.

Outra tema de investigação foi a minimização do efeito agudo de interferência observado quando treinamento de força e treinamento aeróbio são realizados na mesma sessão de treino. De Souza et al. ${ }^{59}$ testaram se o tipo de exercício aeróbio (contínuo ou intervalado) afetava o rendimento em exercícios de resistência de força ou de força máxima realizados subsequentemente. Esse estudo demonstrou que o exercício aeróbio intervalado diminui significantemente a resistência de força e teve uma tendência em diminuir a força máxima. Posteriormente, de SouzA et al..$^{60}$ investigaram o efeito contrário, ou seja, se exercícios de resistência de força e força máxima afetavam agudamente o rendimento de exercícios aeróbios (i.e. contínuo e intervalado) realizados posteriormente na mesma sessão. Os achados indicaram que nenhum dos tipos de exercício de força afetou o rendimento aeróbio. Como os efeitos agudos de interferência em protocolos de treinamento concorrente ainda náo estavam completamente elucidados, SiLva ${ }^{61}$ testou se diferentes volumes de exercício aeróbio contínuo afetavam de forma negativa o desempenho de força. Os achados indicaram que quanto maior o volume do treinamento aeróbio, maior é o efeito agudo de interferência negativa nos exercícios de força realizados posteriormente.

$\mathrm{O}$ efeito da organização da carga de treinamento de força no rendimento de atletas, em particular em testes motores, tem sido tema de inúmeros estudos ao longo das últimas décadas. Os estudos nesta temática se concentraram em duas linhas básicas. A primeira verificou os efeitos de modelos com variaçóes de volume e intensidade do treinamento de força (i.e. periodização linear e periodização ondulada semanal) comparados com um modelo em que o volume e a intensidade do treinamento náo variavam (i.e. treinamento não periodizado). Nesse enfoque, Monteiro et al. ${ }^{62}$ demonstraram que a periodização ondulada semanal produziu maiores ganhos de força do que a periodização linear e o treinamento não periodizado. Contudo, esses achados não foram confirmados por de Souza et al. ${ }^{63}$. Esses autores encontraram que esses três modelos de organização da carga de treinamento produziram ganhos de força similares. A razão para os achados contraditórios ainda não é completamente clara para nós, porém os estudos na segunda linha ajudam na elucidação dessa contradição. Loturco et al. ${ }^{64-66}$ estudaram diferentes modelos de organização do treinamento de força e potência durante um período de treinamento. Os achados indicaram que os diferentes modelos de organização do treinamento produziram melhoras similares no rendimento de diferentes testes motores. Em conjunto, nossos estudos indicam que o modelo de organização da carga de treinamento ao longo do tempo não parece ter um efeito determinante na melhora do rendimento.

Trocando de abordagem, o Grupo também investigou os efeitos de diferentes métodos de treinamento de força na coordenação e no controle de habilidades motoras básicas: o salto vertical e a corrida. LAMAs et al. ${ }^{67}$ verificaram que o treinamento de força e o treinamento de potência não produziram mudanças na estrutura coordenativa do salto vertical, indicando que essa habilidade motora é 
extremamente estereotipada e que o treinamento não pode alterar a sua estrutura coordenativa, como sugerido anteriormente na literatura por BOBBERT e Van Soest ${ }^{68}$. Por outro lado, Roschel et al. ${ }^{69}$ testaram se o treinamento de força e o treinamento de força realizado em plataforma vibratória poderia alterar a rigidez dos membros inferiores durante a corrida. De maneira semelhante aos achados de LAMAs et al. ${ }^{67}$, náo foram encontradas alteraçóes na estrutura do movimento da corrida com os dois modelos de treinamento.

O quarto tópico de investigação do grupo diz respeito aos efeitos do treinamento de força em tarefas técnicas, táticas e estratégicas em modalidades esportivas. CARMO ${ }^{70}$ investigou se o treinamento pliométrico poderia alterar na estratégia de corrida numa prova de $10 \mathrm{~km}$. Os resultados encontrados indicaram que esse treinamento não foi efetivo em alterar a estratégia dos corredores mas aumentou o desempenho deles. Finalmente, Kitamura ${ }^{71}$ testou se o treinamento combinado de força e potência poderia aumentar o rendimento de atletas de voleibol, quando comparado com atletas que apenas realizaram o treinamento técnico/tático. É importante destacar que esse modelo de treinamento foi efetivo em aumentar o rendimento tanto técnico quanto tático das atletas. Esse estudo pode ser considerado importante, pois foi um dos primeiros na literatura a demonstrar a transferência dos efeitos do treinamento físico em açôes técnico/táticas em uma modalidade esportiva coletiva.

Em resumo, os resultados dos estudos conduzidos pelo Grupo de Estudo e Pesquisa em Adaptaçóes Neuromusculares ao Treinamento de Força têm demonstrado a importância da aplicação e combinação de diferentes métodos de treinamento e da organizaçáo da distribuição da carga para a melhora do desempenho em diferentes testes motores. Além disso, o estudo dos efeitos do treinamento de força e potência na coordenação e no controle de habilidades motoras básicas e especialmente nas açóes técnicas e táticas de modalidades esportivas é um tema de destaque que merece maior atenção nas nossas investigações.

\section{Treinamento em modalidades esportivas de combate}

As modalidades esportivas de combate representam elevado percentual de medalhas disputadas nos Jogos Olímpicos (20\% de todas as medalhas de ouro e $21,3 \%$ de todas as medalhas disputadas ${ }^{72}$, além de atraírem grande público e elevadas premiaçôes em suas vertentes profissionais (p.ex., boxe e "Mixed Martial Arts") ${ }^{73}$. Apesar desta representatividade, a quantidade de trabalhos científicos sobre essas modalidades não é táo elevada quanto para outros esportes $^{74}$. É provável que isso se deva à diversidade de estilos (modalidades de domínio, de percussáo ou mistas) e à complexidade das açóes envolvidas nas disputas competitivas. $\mathrm{O}$ processo de organização do treinamento esportivo por si também é bastante complexo e pode ser otimizado se conhecimentos de diferentes origens forem incorporados e integrados ${ }^{75}$, com iniciativas bem sucedidas sendo apoiadas por consórcios de universidades e pesquisadores de diferentes áreas das Ciências do Esporte ${ }^{76}$.

Assim, nesta seção serão apresentados os principais trabalhos conduzidos pelo Grupo de Estudos e Pesquisas em Lutas, Artes Marciais e Modalidades de Combate da EEFE-USP, bem como por grupos de pesquisadores de instituiçóes parceiras, com ênfase àqueles desenvolvidos junto ao PPGEEFE-USP, tendo como foco central as investigaçóes cujos resultados permitam a melhor organização do Treinamento Esportivo.

No contexto das modalidades esportivas de combate, a caracterização física e fisiológica de atletas bem sucedidos em nível internacional parece ser a abordagem mais usual, permitindo inclusive a publicação de revisóes sobre essa temática em modalidades como o boxe $\mathrm{b}^{77}$, a esgrima ${ }^{78}$, o judồ ${ }^{79}$, o karatêt $\hat{e}^{80}$, a luta olímpica ${ }^{81}$ e o taekwondo ${ }^{82}$, apenas para citar aquelas a serem disputadas em Tóquio 2020. Essa abordagem parte do pressuposto de que existem características físicas e de aptidão física que necessitam ser atingidas para o alto desempenho, embora não seja possível identificar como os atletas atingiram tal perfil.

Outro aspecto importante relacionado a estas modalidades, diz respeito à caracterização fisiológica do combate, uma vez que acredita-se que a melhor compreensão da demanda imposta ao atleta durante tal atividade pode ser de grande importância para a seleção dos meios e métodos de treinamento que possam atender mais adequadamente o princípio 
da especificidade e, desta forma, maximizar o desempenho dos atletas. Neste sentido, diferentes trabalhos foram conduzidos com o objetivo de estimar a contribuição energética durante o boxe ${ }^{83}$, a esgrima ${ }^{84}$, o judô ${ }^{85}$, o karatêt $\hat{t}^{86}$ e taekwondo ${ }^{87}$.

No entanto, é importante considerar que, dado o contato constante entre os lutadores durante a competiçáo, estes estudos utilizaram-se de simulações de luta, para que as medidas pudessem ser realizadas. Ainda assim, estes trabalhos permitiram desdobramentos importantes em relação ao uso de agentes ergogênicos sobre a contribuição energética ${ }^{88}$ e desempenho dos atletas ${ }^{89}$, embora não sejam conhecidos trabalhos que tenham analisado o efeito de diferentes tipos de treinamento sobre a contribuição energética durante a luta nas distintas modalidades.

Em decorrência da impossibilidade de mensuração de respostas fisiológicas em situação de competição oficial, alguns estudos objetivaram realizar a análise técnica e tática destas modalidades, o que permitiria inferir sua demanda fisiológica, bem como estruturar mais adequadamente o treinamento técnico e tático destes atletas. Inúmeros trabalhos foram desenvolvidos sobre esta temática, especialmente nas duas últimas décadas, com destaque para investigaçóes sobre a estrutura temporal e fatores discriminantes de desempenho no boxe ${ }^{90-92}$, na

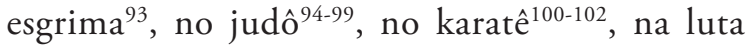
olímpica ${ }^{103}$ e no taekwondo ${ }^{104}$.

Além destas potenciais aplicaçóes, o conhecimento gerado a partir da análise técnica, tática e de estrutura temporal, juntamente com aquele oriundo de investigaçôes sobre a demanda energética da luta, tem permitido a elaboração de processos de avaliação mais específicos. Neste contexto, vale destacar o processo de avaliaçáo física de atletas do boxe ${ }^{105}$, da esgrima ${ }^{106}$, do judôô ${ }^{107-108}$ - incluindo atletas paralímpicos ${ }^{109}$, do karatêt $^{110-114}$ e do taekwondo ${ }^{115-116}$, com utilização de testes específicos - inclusive com a elaboração de tabelas classificatórias ${ }^{117-118}$ e análise discriminante entre diferentes testes ${ }^{119} \mathrm{e} / \mathrm{ou}$ em situaçóes específicas das diferentes modalidades ${ }^{120-122}$.

A compreensão de como treinam os atletas que participaram dos Jogos Olímpicos ${ }^{123}$, de quais competiçóes participam durante o processo de qualificação para esta, com determinação da importância relativa quanto ao posicionamento em "rankings" internacionais ${ }^{124-126} \mathrm{e}$ do intervalo ideal entre estas competiçóes para a maximização do desempenho ${ }^{127}$, permite melhor planejamento do processo de treinamento, além da elaboraçáo de estudos com maior validade ecológica.
Parte destes estudos visou o entendimento sobre como diferentes estímulos agudos afetariam o desempenho em situaçóes específicas destas modalidades, com destaque para estudos que tenham induzido a fadiga prévia ${ }^{128}$ ou investigado a potencialização pós-ativação ${ }^{129-130}$. Adicionalmente, compreender as respostas fisiológicas à sequência de lutas ${ }^{131-132}$, de forma similar ao modo como ocorre em competiçáo, o processo de recuperação entre as lutas ${ }^{133-134}$ e a influência de estratégias nutricionais à sessão de treinamento ${ }^{135}$, são pontos importantes para direcionar o programa de treinamento para o aperfeiçoamento de componentes que influenciem o desempenho competitivo.

Outro ponto importante no cenário atual de investigaçóes sobre Treinamento Esportivo diz respeito ao monitoramento das cargas de treinamento e este aspecto tem sido explorado mais recentemente nas modalidades esportivas de combate ${ }^{136}$, incluindo propostas de métodos de quantificaçáo das cargas de treinamento ${ }^{137}$. O monitoramento da carga interna permite estabelecer inferências sobre como essas cargas podem afetar as respostas imunes, autonômicas e de desempenho em decorrência do modelo de periodizaçáo do treinamento ${ }^{138} \mathrm{ou}$ em fases específicas do treinamento destes atletas ${ }^{139-140}$, em especial quanto ao uso de métodos baseados na percepçáo do esforço da sessão para predizer mudanças no desempenho físico destes atletas ${ }^{141}$.

Por fim, tanto estudos descritivos relacionados ao processo de periodização ${ }^{142}$, quanto estudos experimentais com intervençóes mais curtas, porém permitindo a comparaçáo de diferentes estratégias de treinamento de força ${ }^{143}$ ou de distintos protocolos de treinamento intervalado de alta intensidade ${ }^{144-146}$, sobre o desempenho em testes genéricos e específicos, bem como em situação de luta, têm sido conduzidos. Os resultados destes estudos permitem conceber estratégias mais apropriadas de treinamento para o desenvolvimento de características específicas para o desempenho (p.ex., potência muscular, potência e capacidade aeróbias e anaeróbias). Sem dúvida, a condução de pesquisas comparando diferentes tipos de treinamento ou modelos de periodização e o impacto destas intervençóes no desempenho técnico e tático dos atletas é o desafio a ser enfrentado nos próximos anos.

Assim, é possível constatar que nos últimos 20 anos, os principais tópicos relacionados a modalidades esportivas de combate, com interface com a área de Treinamento Esportivo, envolveram a caracterização da resposta fisiológica a situaçóes específicas destas modalidades, o estabelecimento da 
estrutura temporal e das principais ações executadas em competiçóes oficiais, o monitoramento das cargas de treinamento, o desenvolvimento de testes específicos e a análise de respostas fisiológicas e de desempenho ao treinamento. Estas investigações são decorrentes de dissertaçóes de mestrado e teses de doutorado concluídas e em processo de finalização, todas dentro do PPGEEFE-USP.

\section{Conclusão}

A preocupação com o aperfeiçoamento do rendimento na prática esportiva aborda frequentemente aspectos associados ao desenvolvimento de capacidades motoras, organização e controle do processo de treinamento e métodos/estratégias de preparação. Sabe-se que a prescrição ótima do treinamento passa por um bom entendimento de fatores fisiológicos, biomecânicos, psicológicos e metodológicos os quais permitem a interpretação das respostas do atleta frente às cargas de treinamento e os ajustes necessários aos estímulos oferecidos. Nos últimos anos, um grupo de docentes e discentes do PPGEEFE-USP tem se dedicado ao estudo e ao entendimento dos diferentes aspectos que afetam o processo de treinamento e o rendimento físico-esportivo dos indivíduos tanto em modalidades esportivas de caráter individual quanto coletivo.

Apesar dos evidentes avanços alcançados, um importante desafio permanece para os atuais e principalmente para os futuros pesquisadores na área do Treinamento Esportivo: a maioria dos estudos ainda necessita de uma visão mais integrativa capaz de envolver não somente os aspectos biológicos e organizacionais do rendimento esportivo mas um entendimento do processo de treinamento e do atleta como um todo.

\section{Referências}

1. Borresen J, Lambert MI. The quantification of training load, the training response and the effect on performance. Sports Med. 2009;39:779-95.

2. Lambert MI, Borresen J. Measuring training load in sports. Int J Sports Physiol Perform. 2010;5:406-11.

3. Pires, FO, de-Oliveira, FR. Homenagem à professora Maria Augusta Peduti Dal Molin Kiss. Rev Bras Educ Fís Esporte. 2014;28:533-4.

4. Bueno, S. Associação do polimorfismo da ECA e variáveis fisiológicas determinantes da aptidão aeróbia [dissertação]. São Paulo: Universidade de São Paulo, Escola de Educação Física e Esporte; 2015.

5. Pasqua LA. Associação entre o polimorfismo de nucleotídeo único no gene ACTN3, variáveis fisiológicos e parâmetros neuromusculares relacionados à aptidão aeróbia [dissertação\}. São Paulo: Universidade de São Paulo, Escola de Educação Física e Esporte; 2015.

6. Bouchard C, Lesage R, Lortie G, et al. Aerobic performance in brothers, dizygotic and monozygotic twins. Med Sci Sports Exerc. 1986;18:639-46.

7. Pasqua LA, Bueno S, Artioli GG, et al. Influence of ACTN3 R577X polymorphism on ventilatory thresholds related to endurance performance. J Sports Sci. 2016;34:163-70.

8. Pasqua LA, Bueno S, Matsuda M, et al. The genetics of human running: ACTN3 polymorphism as an evolutionary tool improving the energy economy during locomotion. Ann Hum Biol. 2016;43:255-60.

9. Bueno S, Pasqua LA, de Araújo G, Lima-Silva AE, Bertuzzi R. The association of ACE genotypes on cardiorespiratory variables related to physical fitness in healthy men. PLoS One. 2016;11:e0165310.

10. Silva RC. Impacto de quatro semanas de treinamento intervalado de alta intensidade sobre variáveis fisiológicas associadas à aptidão aeróbia e a estratégia de corrida durante a simulação de uma prova de $5.000 \mathrm{~m}$ [dissertação]. São Paulo: Universidade de São Paulo, Escola de Educação Física e Esporte; 2013.

11. Damasceno MV. Influência do treinamento de força sobre a estratégia de prova e o desempenho de corredores de longa distância em um teste contrarrelógio de 10 km [tese]. São Paulo: Universidade de São Paulo, Escola de Educação Física e Esporte; 2015. 
12. Damasceno MV, Lima-Silva AE, Pasqua LA, et al. Effects of resistance training on neuromuscular characteristics and pacing during 10-km running time trial. Eur J Appl Physiol. 2015;115:1513-22.

13. Brooks GA, Fahey TD, Baldwin KM. Exercise physiology: human bioenergetics and its applications. 4th ed. New York: McGraw-Hill; 2005.

14. Coyle EF. Physical activity as a metabolic stressor. Am J Clin Nutr. 2000;72:512S-20S.

15. Gatti R, De Palo EF. An update: salivary hormones and physical exercise. Scand J Med Sci Sports. 2011;21:157-69.

16. Moreira A, Arsati F, de Oliveira Lima Arsati YB, da Silva DA, de Araujo VC. Salivary cortisol in top-level professional soccer players. Eur J Appl Physiol. 2009;106:25-30.

17. Bourdon PC, Cardinale M, Murray A, et al. Monitoring athlete training loads: consensus statement. Int J Sports Physiol Perform. 2017;12:S2161-70.

18. Moreira A, Arsati F, Cury PR, Franciscon C, Oliveira PR, Araújo VC. Salivary immunoglobulin A responses to a match in top-level Brazilian soccer players. J Strength Cond Res. 2009;23:1968-73.

19. Moreira A, Arsati F, de Oliveira Lima-Arsati YB, de Freitas CG, de Araújo VC. Salivary immunoglobulin A responses in professional top-level futsal players. J Strength Cond Res. 2011;25:1932-936.

20. Moreira A, Bacurau RF, Napimoga MH, et al. Salivary IL-21 and IgA responses to a competitive match in elite basketball players. Biol Sport. 2013;30:243-47.

21. Moreira A, McGuigan MR, Arruda AF, Freitas CG, Aoki MS. Monitoring internal load parameters during simulated and official basketball matches. J Strength Cond Res. 2012;26:861-6.

22. Moreira A, Crewther B, Freitas CG, Arruda AF, Costa EC, Aoki MS. Session RPE and salivary immune-endocrine responses to simulated and official basketball matches in elite young male athletes. J Sports Med Phys Fitness. 2012;52:682-7.

23. Moreira A, Franchini E, de Freitas CG, et al. Salivary cortisol and immunoglobulin A responses to simulated and official Jiu-Jitsu matches. J Strength Cond Res. 2012;26:2185-91.

24. Freitas CG, Aoki MS, Arruda AF, Franciscon C, Moreira A. Monitoring salivary immunoglobulin A responses to official and simulated matches in elite young soccer players. J Hum Kinet. 2016;53:107-15.

25. Moreira A, Freitas CG, Nakamura FY, Drago G, Drago M, Aoki MS. Effect of match importance on salivary cortisol and immunoglobulin A responses in elite young volleyball players. J Strength Cond Res. 2013;27:202-7.

26. Arruda AF, Aoki MS, Freitas CG, et al. Influence of competition playing venue on the hormonal responses, state anxiety and perception of effort in elite basketball athletes. Physiol Behav. 2014;10;130:1-5.

27. Arruda AF, Aoki MS, Miloski B, Freitas CG, Moura NR, Moreira A. Playing match venue does not affect resting salivary steroids in elite Futsal players. Physiol Behav. 2016;1;155:77-82.

28. Marcelino PR, Aoki, MS, Arruda AFS, Freitas CG, Mendez-Villanueva A, Moreira A. Does small-sided-games' court area influence metabolic, perceptual, and physical performance parameters of young elite basketball players? Biol Sport. 2016;33:37-42.

29. Moreira A, Aoki MS, Carling C, et al. Temporal changes in technical and physical performances during a small-sided game in elite youth soccer players. Asian J Sports Med. 2016;7:e35411.

30. Rodrigues Lopes RA. Desempenho técnico em jogos reduzidos em jovens jogadores de futebol: uma abordagem longitudinal [dissertação]. São Paulo: Universidade de São Paulo, Escola de Educação Física e Esporte; 2017.

31. Moreira A, Bilsborough JC, Sullivan CJ, Ciancosi M, Aoki MS, Coutts AJ. Training periodization of professional Australian football players during an entire Australian Football League season. Int J Sports Physiol Perform. 2015;10:566-71.

32. Moreira A, Kempton T, Aoki MS, Sirotic AC, Coutts AJ. The impact of 3 different-length between-matches microcycles on training loads in professional rugby league players. Int J Sports Physiol Perform. 2015;10:767-73.

33. Mujika I. Intense training: the key to optimal performance before and during the taper. Scand J Med Sci Sport. 2010;Suppl 2:24-31.

34. Freitas CG, Aoki MS, Franciscon CA, Arruda AF, Carling C, Moreira A. Psychophysiological responses to overloading and tapering phases in elite young soccer players. Pediatr Exerc Sci. 2014;26:195-202.

35. Dias BM. O efeito da estratégia de periodização baseada na intensificação e tapering nas respostas hormonais, comportamentais, perceptuais e de desempenho em jovens jogadores de basquetebol [tese]. São Paulo: Universidade de São Paulo, Escola de Educação Física e Esporte; 2015.

36. Moreira A, Arsati F, Lima-Arsati YBO, Simões AC, de Araújo, VC. Monitoring stress tolerance and occurrences of upper respiratory illness in basketball players by means of psychometric tools and salivary biomarkers. Stress Health. 2011;3:e166-72. 
37. Nunes JA, Moreira A, Crewther BT, Nosaka K, Viveiros L, Aoki MS. Monitoring training load, recovery-stress state, immune-endocrine responses, and physical performance in elite female basketball players during a periodized training program. J Strength Cond Res. 2014;28:2973-80.

38. Miloski B, Aoki MS, de Freitas CG, et al. Does testosterone modulate mood states and physical performance in young basketball players? J Strength Cond Res. 2015;29:2474-81.

39. Aoki MS, Ronda LT, Marcelino PR, et al. Monitoring training loads in professional basketball players engaged in a periodized training program. J Strength Cond Res. 2017;31:348-58.

40. Moraes H, Aoki MS, Freitas CG, Arruda A, Drago G, Moreira A. SIgA response and incidence of upper respiratory tract infections during intensified training in youth basketball players. Biol Sport. 2017;34:49-55.

41. Moreira A, Nakamura FY, Cavazzoni PB, Gomes JH, Martignago P. O efeito da intensificação do treinamento na percepção de esforço da sessão e nas fontes e sintomas de estresse em jogadores jovens de basquetebol. Rev Educ Fís. 2010;21:287-96.

42. Moreira A, Moura NR, Coutts A, Costa EC, Kempton T, Aoki MS. Monitoring internal training load and mucosal immune responses in futsal athletes. J Strength Cond Res. 2013;27:1253-9.

43. Gomes RV, Moreira A, Lodo L, Nosaka K, Coutts AJ, Aoki MS. Monitoring training loads, stress, immune-endocrine responses and performance in tennis players. Biol Sport. 2013;30:173-80.

44. Antualpa K, Aoki MS, Moreira A. Salivary steroids hormones, well-being, and physical performance during an intensification training period followed by a tapering period in youth rhythmic gymnasts. Physiol Behav. 2017;179:1-8.

45. Moreira A, Mortatti AL, Arruda AF, Freitas CG, de Arruda M, Aoki MS. Salivary IgA response and upper respiratory tract infection symptoms during a 21-week competitive season in young soccer players. J Strength Cond Res. 2014;28:467-73.

46. Moreira A, Arsati F, Cury PR, et al. The impact of a 17-day training period for an international championship on mucosal immune parameters in top-level basketball players and staff members. Eur J Oral Sci. 2008;116:431-7.

47. Moreira A, Mortatti A, Aoki M, Arruda A, Freitas C, Carling C. Role of free testosterone in interpreting physical performance in elite young Brazilian soccer players. Pediatr Exerc Sci. 2013;25:186-97.

48. Arruda AF, Aoki MS, Freitas CG, Spigolon LM, Franciscon C, Moreira A. Testosterone concentration and lower limb power over an entire competitive season in elite young soccer players. J Strength Cond Res. 2015;29:3380-5.

49. Aoki MS, Arruda AFS, Freitas CG, et al. Monitoring training loads, mood states, and jump performance over two periodized training mesocycles in elite young volleyball players. Int J Sports Sci Coach. 2017;12:130-7.

50. Carling C, Gregson W, McCall A, Moreira A, Wong del P, Bradley PS. Match running performance during fixture congestion in elite soccer: research issues and future directions. Sports Med. 2015;45:605-13.

51. Arruda AF, Carling C, Zanetti V, Aoki MS, Coutts AJ, Moreira A. Effects of a very congested match schedule on bodyload impacts, accelerations, and running measures in youth soccer players. Int J Sports Physiol Perform. 2015;10:248-52.

52. Moreira A, Bradley P, Carling C, et al. Effect of a congested match schedule on immune-endocrine responses, technical performance and session-RPE in elite youth soccer players. J Sports Sci. 2016;34:2255-61.

53. Tricoli V, Lamas L, Carnevale R, Ugrinowitsch C Short-term effects on lower-body functional power development: weightlifting vs. vertical jump training programs. J Strength Cond Res. 2005;19:433-7.

54. Lamas L, Drezner R, Tricoli V, Ugrinowitsch C Efeito de dois métodos de treinamento no desenvolvimento da força máxima e da potência muscular de membros inferiores. Rev Bras Educ Fís Esporte. 2008;22:235-45.

55. Kobal R, Loturco I, Barroso R, et al. Effects of different combinations of strength, power, and plyometric training on the physical performance of elite young soccer players. J Strength Cond Res. EPub 2016 Aug 18.

56. Batista MAB, Ugrinowitsch C, Roschel H, Lotufo R, Ricard MD, Tricoli V Intermittent exercise as a conditioning activity to induce postactievation potentiation. J Strength Cond Res. 2007;21:837-40.

57. Lamas L, Tricoli V, Batista M, Ugrinowitsch C Efeito agudo da vibração sobre o desempenho do agachamento em alta velocidade e salto vertical. Rev Bras Cinean Desemp Hum. 2007;12:401-7.

58. Batista MAB, Roschel H, Barroso R, Ugrinowitsch C, Tricoli V Influence of strength training background on postactivation potentiation response. J Strength Cond Res. 2011;25:2496-502.

59. de Souza EO, Tricoli V, Franchini E, Paulo AC, Regazzini M, Ugrinowitsch, C Acute effect of two aerobic exercise modes on maximum strength and strength endurance. J Strength Cond Res. 2007;21:1286-90.

60. de Souza EO, Caineli Rosa LF, Pires, FO et al. The acute effects of varying strength exercises bouts on $5 \mathrm{~km}$ running. J Sports Sci Med. 2011;10:565-70.

61. Silva NR Efeito agudo de exercícios aeróbios com diferentes volumes no desempenho de força em indivíduos fisicamente ativos. [dissertação]. São Paulo: Universidade de São Paulo, Escola de Educação Física e Esporte; 2015. 
62. Monteiro AG, Aoki MS, Evangelista AL, et al. Nonlinear periodization maximizes strength gains in split resistance training routines. J Strength Cond Res. 2009;23:1321-6.

63. Souza EO, Ugrinowitsch C, Tricoli V, et al. Early adaptations to six weeks of non-periodized and periodized strength training regimens in recreational males. J Sports Sci Med. 2014;13:604-9.

64. Loturco I, Ugrinowitsch C, Roschel H, Tricoli V, Badillo JJG Distinct temporal organizations of the strength- and power-training loads produce similar performance improvements. J Strength Cond Res. 2012;27:188-94.

65. Loturco I, Ugrinowitsch C, Roschel H, Tricoli V, Gonzalez-Badillo JJ Training at the optimum power zone produces similar performance improvements to traditional strength training. J Sports Sci Med. 2013;12:109-15.

66. Loturco I, Ugrinowitsch C, Tricoli V, Pivetti B, Roschel H Different loading schemes in power training during the preseason promote similar performance improvements in Brazilian elite soccer players. J Strength Cond Res. 2013;27:1791-7.

67. Lamas L, Ugrinowitsch C, Rodacki A, et al. Effects of strength and power training on neuromuscular adaptations and jumping movement pattern and performance. J Strength Cond Res. 2012;26:3335-44.

68. Bobbert MF, van Soest AJ, Effects of muscle strengthening on vertical jump height: a simulation study." Med Sci Sports Exerc. 1994;26:1012-20.

69. Roschel H, Barroso R, Tricoli V, et al. Effects of strength training associated with whole-body vibration training on running economy and vertical stiffness. J Strength Cond Res. 2015;29:2215-20.

70. Carmo EC Efeito da economia de corrida sobre s estratégia de prova utilizada durante uma corrida de $10 \mathrm{~km}$ [tese]. São Paulo: Universidade de São Paulo, Escola de Educação Física e Esporte; 2014.

71. Kitamura KRP Efeito das alterações induzidas pelo treinamento combinado de força e potência nas ações técnicotática-estratégicas de jogadoras de voleibol. [dissertação]. São Paulo: Universidade de São Paulo, Escola de Educação Física e Esporte; 2013.

72. Franchini E, Takito MY. Home advantage in combat sports during the Olympic Games. Sport Sci Health. 2016; 12:287-90.

73. Franchini E. Born to fight? Genetics and combat sports. Rev Artes Marciales Asiát. 2014;9:1-8.

74. Franchini E, Del Vecchio FB. Estudos em modalidades esportivas de combate: estado da arte. Rev Bras Educ Fis Esporte. 2011;25:67-81.

75. Smith DJ. A framework for understanding the training process leading to elite performance. Sports Med. 2003; 33:1103-26.

76. Rees T, Hardy L, Güllich A, et al. The Great British Medalists Project: a review of current knowledge on the development of the world's best sporting talent. Sports Med. 2016;46:1041-58.

77. Chaabene H, Hachana Y, Tabben M, et al. Amateur boxing: physical and physiological attributes. Sports Med. 2015;45:337-52.

78. Turner A, James N, Dimitriou L, et al. Determinants of Olympic fencing performance and implications for strength and conditioning training. J Strength Cond Res. 2014;28:3001-11.

79. Franchini E, Matsushigue KA, Vecchio FB, Artioli GG. Physiological profiles of elite judo athletes. Sports Med. 2011;41:147-66.

80. Chaabene H, Hachana Y, Franchini E, Makaouer B, Chamari K. Physical and physiological profile of elite karate athletes. Sports Med. 2012;42:829-43.

81. Chaabene H, Negra Y, Bouguezzi R, et al. Physical and physiological profile of wrestler athletes. J Strength Cond Res. 2017;31:1411-42.

82. Bridge C, Santos JFS, Chaabene H, Pieter W, Franchini E. Physical and physiological profiles of taekwondo athletes. Sports Med. 2014;44:713-33.

83. Davis P, Leithäuser RM, Beneke R. The energetics of semicontact 3 x 2-min amateur boxing. Int J Sports Physiol Perform. 2014;9:233-9.

84. Milia R, Roberto S, Pinna M, et al. Physiological responses and energy expenditure during competitive fencing. Appl Physiol Nutr Metab. 2014;39:324-8.

85. Julio UF, Panissa VL, Esteves JV, Cury RL, Agostinho MF, Franchini E. Energy system contributions to simulated judo matches. Int J Sports Physiol Perform. 2016; Oct 13:1-24. [Epub ahead of print].

86. Beneke R, Beyer T, Jachner C, Erasmus J, Hütler M. Energetics of karate kumite. Eur J Appl Physiol. 2004;92:518-23.

87. Campos FA, Bertuzzi R, Dourado AC, Santos VG, Franchini E. Energy demands in taekwondo athletes during combat simulation. Eur J Appl Physiol. 2012;112:1221-8.

88. Lopes-Silva JP, Silva Santos JF, Branco BH, et al. Caffeine ingestion increases estimated glycolytic metabolism during taekwondo combat simulation but does not improve performance or parasympathetic reactivation. PLoS One. 2015;10:e0142078. 
89. Andrade Kratz C, de Salles Painelli V, de Andrade Nemezio KM, et al. Beta-alanine supplementation enhances judorelated performance in highly-trained athletes. J Sci Med Sport. 2017;20:403-8.

90. Davis P, Benson PR, Pitty JD, Connorton AJ, Waldock R. The activity profile of elite male amateur boxing. Int J Sports Physiol Perform. 2015;10:53-7.

91.Davis P, Connorton A, Driver S, Anderson S, Waldock R. The activity profile of elite male amateur boxing following the 2013 rule changes. J Strength Cond Res. 2017 Feb 23; [Epub ahead of print].

92. Slimani M, Chaabène H, Davis P, Franchini E, Cheour F, Chamari K. Performance aspects and physiological responses in male amateur boxing competitions: a brief review. J Strength Cond Res. 2017;31:1132-41.

93. Wylde MJ, Tan FHY, O’Donoghue PG. A time-motion analysis of elite women's foil fencing. Int J Perform Anal Sport. 2013;13:365-76.

94. Calmet M, Miarka B, Franchini E. Modeling approaches of grasps in judo competition contests. Int J Permrf Anal Sport. 2010;10:229-40.

95. Franchini E, Sterkowicz S, Meira CM, Gomes FR, Tani G. Technical variation in a sample of high level judo players. Percept Mot Skills. 2008;106:859-69.

96. Ibañez JC, Femia O, Stankovic N, Franchini E, Molina RE. Analysis of kumi-kata grip laterality and throwing side on attack effectiveness and combat result in elite judokas. Int J Perform Anal Sport. 2014;14:138-47.

97. Marcon G, Franchini E, Jardim JR, Barros Neto TL, Structural analysis of action and time in sports: judo. J Quant Anal Sports. 2010;6:1-13.

98. Miarka B, Panissa VL, Julio UF, Del Vecchio FB, Calmet M, Franchini E. A comparison of time-motion performance between age groups in judo matches. J Sports Sci. 2012;30:899-905.

99. Molina RE, Ibáñez JC, Franchini E, Femia O, Stankovic N. The impact of penalties on subsequent attack effectiveness and combat outcome among high elite judo competitors. Int J Perform Anal Sport. 2014;14:946-54.

100. Chaabène H, Franchini E, Miarka B, Selmi MA, Mkaouer B, Chamari K. Time-motion analysis and physiological responses to karate official combat sessions: is there a difference between winners and defeated karatekas? Int J Sports Physiol Perform. 2014;9:302-8.

101. Chaabene H, Hellara I, Ghali FB, et al. Physiological stress and performance analysis to karate combat. J Sports Med Phys Fitness. 2016;56:1125-31.

102. Tabben M, Coquart J, Chaabène H, Franchini E, Ghoul N, Tourny C. Time-motion, tactical and technical analysis in top-level karatekas according to gender, match outcome and weight categories. J Sports Sci. 2015;33:841-9.

103. Nilsson J, Csergö S, Gullstrand L, Tveit P, Refsnes PE. Work-time profile, blood lactate concentration and rating of perceived exertion in the 1998 Greco-Roman Wrestling World Championship. J Sports Sci. 2002;20:939-45.

104. Santos VG, Franchini E, Lima-Silva AE. Relationship between attack and skipping in taekwondo contests. J Strength Cond Res. 2011;25:1743-51.

105. Loturco I, Nakamura FY, Artioli GG, et al. Strength and power qualities are highly associated with punching impact in elite amateur boxers. J Strength Cond Res. 2016;30:109-16.

106. Weichenberger M, Liu Y, Steinacker JM. A test for determining endurance capacity in fencers. Int J Sports Med. 2012;33:48-52.

107. Almansba R, Franchini E, Sterkowicz S. Uchi-komi avec charge, une approche physiologique d?un nouveau test spécifique au judo. Sci Sports. 2007;22:216-23.

108. Franchini E, Sterkowicz S, Szmatlan-Gabrys U, Gabrys T, Garnys M. Energy system contributions to the special judo fitness test. Int J Sports Physiol Perform. 2011;6:334-43.

109. Loturco I, Nakamura FY, Winckler C, et al. strength-power performance of visually impaired Paralympic and Olympic judo athletes from the Brazilian national team: a comparative study. J Strength Cond Res. 2017;31:743-9.

110. Loturco I, Artioli GG, Kobal R, Gil S, Franchini E. Predicting punching acceleration from selected strength and power variables in elite karate athletes: a multiple regression analysis. J Strength Cond Res. 2014;28:1826-32.

111. Loturco I, Nakamura FY, Lopes-Silva JP, Santos JFS, Pereira LA, Franchini E. Physical and physiological traits of a double world karate champion and responses to a simulated kumite bout: a case study. Int J Sports Sci Coach. 2017; 12:138-47.

112. Roschel H, Batista M, Monteiro R, et al. Association between neuromuscular tests and kumite performance on the brazilian karate national team. J Sports Sci Med. 2009;8:20-4.

113. Sterkowicz S, Franchini E. Testing motor fitness in karate. Arch Budo. 2009;5:29-34.

114. Tabben M, Coquart J, Chaabène H, Franchini E, Chamari K, Tourny C. Validity and reliability of new karate-specific aerobic test for karatekas. Int J Sports Physiol Perform. 2014;9:953-8. 
115. Santos JF, Valenzuela TH, Franchini E. Can different conditioning activities and rest intervals affect the acute performance of taekwondo turning kick? J Strength Cond Res. 2015;29:1640-7.

116. Santos JFS, Franchini E. Is frequency speed of kick test responsive to training? A study with taekwondo athletes. Sport Sci Health. 2016;2:377-82.

117. Branco BHM, Diniz E, Santos JFS, Shiroma SA, Franchini E Normative tables for the dynamic and isometric judogi chin-up tests for judo athletes. Sport Sci Health. 2017;13:47-53.

118. Franchini E, Vecchio FB, Sterkowicz S. A special judo fitness test classificatory table. Arch Budo. 2009;5:127-9.

119. Tavra M, Franchini E, Krstulovic S. Discriminant and factorial validity of judo-specific tests in female athletes. Arch Budo. 2016;12:93-9.

120. Chaabène H, Mkaouer B, Franchini E, et al. Physiological responses and performance analysis difference between official and simulated karate combat conditions. Asian J Sports Med. 2014;5:21-9.

121. Franchini E, Panissa VL, Julio UF. Physiological and performance responses to intermittent uchi-komi in judo. J Strength Cond Res. 2013;27:1147-55.

122. Tabben M, Chaabène H, Franchini E, Tourny C, Chamari K, Coquart J. The influence of karate practice level and sex on physiological and perceptual responses in three modern karate training modalities. Biol Sport. 2014;31:201-7.

123. Franchini E, Takito MY. Olympic preparation in Brazilian judo athletes: description and perceived relevance of training practices. J Strength Cond Res. 2014;28:1606-12.

124. Franchini E, Julio UF. The judo world ranking list and the performances in the 2012 London Olympics. Asian J Sports Med. 2015;6:e24045.

125. Julio UF, Panissa VL, Miarka B, Takito MY, Franchini E. Home advantage in judo: a study of the world ranking list. J Sports Sci. 2013;31:212-8.

126. Guilheiro LM, Franchini E. Be seeded or not be seeded? A study with Olympic judo athletes. J Exerc Rehab. 2017;13:148-52.

127. Franchini E, Takito MY, da Silva RM, Shiroma SA, Wicks L, Julio UF. Optimal interval for success in Judo World Ranking competitions. Int J Sports Physiol Perform. 2016 Sep 15;[Epub ahead of print].

128. Sant'Ana J, Franchini E, da Silva V, Diefenthaeler F. Effect of fatigue on reaction time, response time, performance time, and kick impact in taekwondo roundhouse kick. Sports Biomech. 2017;16:201-9.

129. Miarka B, Del Vecchio FB, Franchini E. Acute effects and postactivation potentiation in the Special Judo Fitness Test. J Strength Cond Res. 2011;25:427-31.

130. Santos JFS, Herrera T, Mota GR, Franchini E. Influence of half-squat intensity and volume on the subsequent countermovement jump and frequency speed of kick test performance in taekwondo athletes. Kinesiology. 2016; 48:95-102.

131. Bonitch-Góngora JG1, Bonitch-Domínguez JG, Padial P, Feriche B. The effect of lactate concentration on the handgrip strength during judo bouts. J Strength Cond Res. 2012;26:1863-71.

132. Detanico D, Dal Pupo J, Franchini E, Dos Santos SG. Effects of successive judo matches on fatigue and muscle damage markers. J Strength Cond Res. 2015;29:1010-6.

133. Franchini E, Takito MY, Nakamura FY, Matsushigue KA, Kiss MAPDM. Effects of recovery type after a judo combat on blood lactate removal and on performance in an intermittent anaerobic task. J Sports Med Phys Fitness. 2003;43:424-31.

134. Franchini E, Bertuzzi RCM, Takito MY, Kiss MA. Effects of recovery type after a judo match on blood lactate and performance in specific and non-specific judo tasks. Eur J Appl Physiol. 2009;107:377-83.

135. Brito CJ, Gatti K, Mendes EL, et al. Carbohydrate intake and immunosuppression during judo training. Med Sport. 2011;64:393-408.

136. Milanez V, Lima M, Perandini L, Gonçalves C, Franchini E. Avaliação e comparação das respostas da percepção subjetiva de esforço e concentração de lactato em uma competição oficial de karate. Rev Educ Fis/UEM. 2011;22:57-64.

137. Morales J, Franchini E, Garcia-Massó X, Solana-Tramunt M, Buscà B, González LM. The work endurance recovery method for quantifying training loads in judo. Int J Sports Physiol Perform. 2016;11:913-9.

138. Agostinho MF, Moreira A, Julio UF, et al. Monitoring internal training load and salivary immuneendocrine responses during an annual judo training periodization. J Exerc Rehab. 2017;13:68-75.

139. Nakamura FY, Pereira LA, Abad CC, Franchini E, Loturco I. Cardiac autonomic and neuromuscular responses during a karate training camp before the 2015 Pan American Games: a case study with the Brazilian National Team. Int J Sports Physiol Perform. 2016;11:833-7.

140. Branco BHM, Lopes-Silva JP, da Silva Santos JF, Julio UF, Panissa VLG, Franchini E. Monitoring training during four weeks of three different modes of high-intensity interval training in judo athletes. Arch Budo. 2017;13:51-60. 
Tricoli VAA, et al.

141. Agostinho MF, Philippe AG, Marcolino GS, et al. Perceived training intensity and performance changes quantification in judo. J Strength Cond Res. 2015;29:1570-7.

142. Franchini E, Vecchio FB, Julio UF, Mtaheus L, Candau R. Specificity of performance adaptations to a periodized judo training program. Rev Andaluza Med Deporte. 2015;8:67-72.

143. Franchini E, Branco BM, Agostinho MF, Calmet M, Candau R. Influence of linear and undulating strength periodization on physical fitness, physiological, and performance responses to simulated judo matches. J Strength Cond Res. 2015;29:358-67.

144. Bonato M, Rampichini S, Ferrara M, et al. Aerobic training program for the enhancements of HR and VO2 offkinetics in elite judo athletes. J Sports Med Phys Fitness. 2015;55:1277-84.

145. Franchini E, Julio UF, Panissa VL, Lira FS, Gerosa-Neto J, Branco BH. High-intensity intermittent training positively affects aerobic and anaerobic performance in judo athletes independently of exercise mode. Front Physiol. 2016;28;7:268.

146. Franchini E, Julio UF, Panissa VLG, Lira FS, Agostinho MF, Branco BHM. Short-term low-volume high-intensity intermittent training improves judo-specific performance. Arch Budo. 2016;12:219-29.

ENDEREÇO

Valmor Alberto Augusto Tricoli

Escola de Educação Física e Esporte - USP

Av. Prof. Mello Moraes, 65

05508-030 - São Paulo - SP - BRASIL

e-mail: vtricoli@usp.br

154 • Rev Bras Educ Fís Esporte, (São Paulo). 2017 Ago; 31(N esp):139-54. 